\title{
Atomic-scale electronic structure of the cuprate $d$-symmetry form factor density wave state
}

\author{
M. H. Hamidian ${ }^{1,2 \dagger}$, S. D. Edkins ${ }^{1,3 \dagger}$, Chung Koo Kim ${ }^{4}$, J. C. Davis ${ }^{1,3,4,5 \star}{ }^{4}$, A. P. Mackenzie ${ }^{3,6}$, H. Eisaki ${ }^{7}$, \\ S. Uchida ${ }^{8}$, M. J. Lawler ${ }^{1,9}$, E.-A. Kim', S. Sachdev ${ }^{2,10}$ and K. Fujita ${ }^{4}$
}

Research on high-temperature superconducting cuprates is at present focused on identifying the relationship between the classic 'pseudogap' phenomenon ${ }^{1,2}$ and the more recently investigated density wave state ${ }^{3-13}$. This state is generally characterized by a wavevector $Q$ parallel to the planar $\mathrm{Cu}-\mathrm{O}-\mathrm{Cu}$ bonds ${ }^{4-13}$ along with a predominantly $d$-symmetry form factor ${ }^{14-16}$ (dFF-DW). To identify the microscopic mechanism giving rise to this state ${ }^{17-29}$, one must identify the momentum-space states contributing to the dFF-DW spectral weight, determine their particle-hole phase relationship about the Fermi energy, establish whether they exhibit a characteristic energy gap, and understand the evolution of all these phenomena throughout the phase diagram. Here we use energy-resolved sublattice visualization ${ }^{14}$ of electronic structure and reveal that the characteristic energy of the dFF-DW modulations is actually the 'pseudogap' energy $\Delta_{1}$. Moreover, we demonstrate that the dFF-DW modulations at $E=-\Delta_{1}$ (filled states) occur with relative phase $\pi$ compared to those at $E=\Delta_{1}$ (empty states). Finally, we show that the conventionally defined dFF-DW Q corresponds to scattering between the 'hot frontier' regions of momentum-space beyond which Bogoliubov quasiparticles cease to exist ${ }^{30-32}$. These data indicate that the cuprate dFF-DW state involves particle-hole interactions focused at the pseudogap energy scale and between the four pairs of 'hot frontier' regions in momentum space where the pseudogap opens.

A conventional 'Peierls' charge density wave (CDW) in a metal results from particle-hole interactions which open an energy gap at specific regions of $\mathbf{k}$-space that are connected by a common wavevector $\mathbf{Q}$. This generates a modulation in the density of free charge at $\mathbf{Q}$ along with an associated modulation of the crystal lattice parameters. Such CDW states are now very well known ${ }^{33}$. In principle, a density wave modulating at $\mathbf{Q}$ can also exhibit a 'form factor' (FF) with different possible symmetries ${ }^{34,35}$ (see Supplementary Section 1). This is relevant to the high-temperature superconducting cuprates because numerous researchers have recently proposed that the 'pseudogap' regime ${ }^{1,2}$ (PG in Fig. 1a) contains an unconventional density wave with a $d$-symmetry form factor ${ }^{17-29}$. The basic phenomenology of such a state is that intraunit-cell (IUC) symmetry breaking renders the $O_{x}$ and $O_{y}$ sites within each $\mathrm{CuO}_{2}$ unit-cell electronically inequivalent, and that this inequivalence is then modulated periodically at wavevector $\mathbf{Q}$ parallel to $(1,0) ;(0,1)$. The real-space (r-space) schematic of such a $d$-symmetry FF density wave (dFF-DW) at $\mathbf{Q}_{x}$, as shown in Fig. 1b, exemplifies periodic modulations at the $O_{x}$ sites that are $\pi$ out of phase with those at the $O_{y}$ sites. Such a state is then described by $A(\mathbf{r})=D(\mathbf{r}) \cos \left(\phi(\mathbf{r})+\phi_{0}(\mathbf{r})\right)$, where $A(\mathbf{r})$ represents whatever is the modulating electronic degree of freedom, $\phi(\mathbf{r})=\mathbf{Q}_{x} \cdot \mathbf{r}$ is the DW spatial phase at location $\mathbf{r}, \phi_{0}(\mathbf{r})$ represents disorder related spatial phase shifts, and $D(\mathbf{r})$ is the magnitude of the $d$-symmetry form factor ${ }^{14,21,23}$. To distinguish between the various microscopic mechanisms proposed for the $\mathbf{Q}=(Q, 0) ;(0, Q)$ dFF-DW state of cuprates $^{17-29}$, it is essential to establish its atomic-scale phenomenology, including the momentum space (k-space) eigenstates contributing to its spectral weight, the relationship (if any) between modulations occurring above and below the Fermi energy, whether the modulating states in the DW are associated with a characteristic energy gap, and how the dFF-DW evolves with doping.

To visualize such phenomena directly as in Fig. 1c, we use sublattice-phase-resolved imaging of the electronic structure $^{14}$ of the $\mathrm{CuO}_{2}$ plane. Both the scanning tunnelling microscope (STM) tip-sample differential tunnelling conductance $\mathrm{d} I / \mathrm{d} V(\mathbf{r}, E=\mathrm{eV}) \equiv g(\mathbf{r}, E)$ and the tunnel-current $I(\mathbf{r}, E)$ are measured at bias voltage $V=E / e$ and with sub-unitcell spatial resolution. Because the density of electronic states $N(\mathbf{r}, E)$ is related to the differential conductance as $g(\mathbf{r}, E) \propto\left[e I_{s} / \int_{0}^{e V_{s}} N\left(\mathbf{r}, E^{\prime}\right) \mathrm{d} E^{\prime}\right] N(\mathbf{r}, E)$, with $I_{s}$ and $V_{s}$ being arbitrary parameters and the denominator $\int_{0}^{\mathrm{eV}_{\mathrm{s}}} N\left(\mathbf{r}, E^{\prime}\right) \mathrm{d} E^{\prime}$ unknown, valid imaging of $N(\mathbf{r}, E)$ is challenging (Supplementary Section 2). However, one can suppress these serious systematic 'set-point' errors by using $R(\mathbf{r}, E)=I(\mathbf{r}, E) / I(\mathbf{r},-E)$ or $Z(\mathbf{r}, E)=g(\mathbf{r}, E) / g(\mathbf{r},-E)$; this allows distances, wavelengths and spatial phases of electronic structure to be measured accurately. The unprocessed $g(\mathbf{r}, E)$ acquired for and analysed in this paper are measured over very large fields of view (to achieve high phase precision in Fourier analysis), simultaneously maintain deeply sub-unit-cell precision measurements in $\mathbf{r}$ (to achieve high precision in sublattice segregation), and are taken over a wide range of energies $E$ with fine energy spacing, so that energy dependences of $d$-symmetry FF modulations may be accurately determined. We then calculate each sublattice-phase-resolved $Z(\mathbf{r}, E)$ image and separate it into three: the first, $\mathrm{Cu}(\mathbf{r})$, contains

\footnotetext{
${ }^{1}$ LASSP, Department of Physics, Cornell University, Ithaca, New York 14853, USA. ${ }^{2}$ Department of Physics, Harvard University, Cambridge, Massachusetts 02138, USA. ${ }^{3}$ School of Physics and Astronomy, University of St. Andrews, Fife KY16 9SS, Scotland. ${ }^{4}$ CMPMS Department, Brookhaven National Laboratory, Upton, New York 11973, USA. ${ }^{5}$ Kavli Institute at Cornell for Nanoscale Science, Cornell University, Ithaca, New York 14853 , USA. ${ }^{6}$ Max-Planck Institute for Chemical Physics of Solids, D-01187 Dresden, Germany. ${ }^{7}$ Institute of Advanced Industrial Science and Technology, Tsukuba, Ibaraki 305-8568, Japan. ${ }^{8}$ Department of Physics, University of Tokyo, Bunkyo-ku, Tokyo 113-0033, Japan. ${ }^{9}$ Department of Physics and Astronomy, Binghamton University, Binghamton, New York 13902, USA. ${ }^{10}$ Perimeter Institute for Theoretical Physics, Waterloo, Ontario N2L 2Y5, Canada. ${ }^{\dagger}$ These authors contributed equally to this work. *e-mail: jcseamusdavis@gmail.com
} 

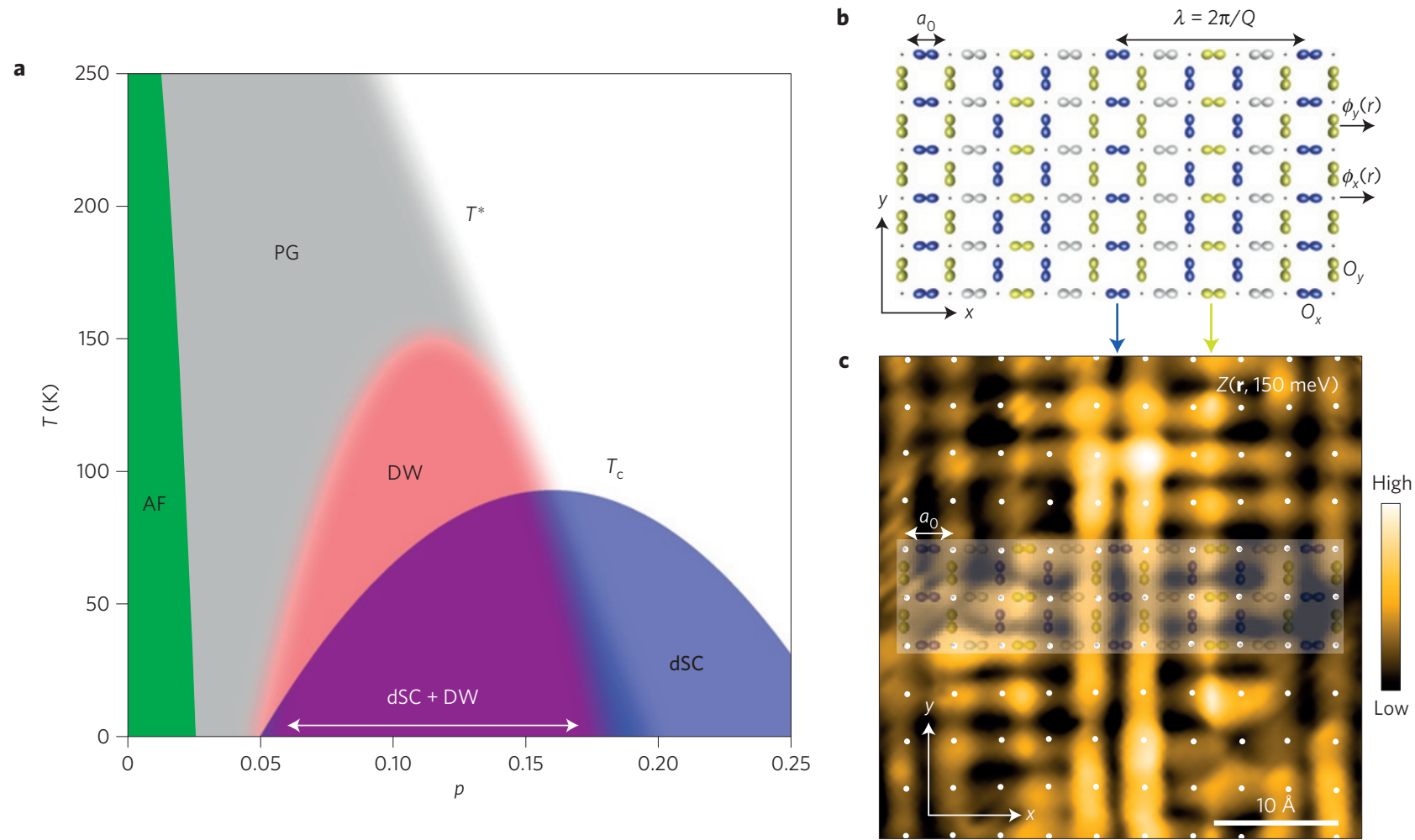

Figure $1 \mid d$-symmetry form factor density wave in the cuprate pseudogap phase. a, Schematic phase diagram of hole-doped cuprates. The pseudogap regime has been identified by, for example, suppression of uniform magnetic susceptibility and electronic specific heat, and the appearance of a truncated Fermi surface, referred to as the 'Fermi arc' ${ }^{\prime 1,2}$. The dome-shaped region of $d$-symmetry Cooper-paired high-temperature superconductivity is universally accepted. More recently, an unusual density wave state has been detected by bulk probes ${ }^{4-13}$ in the region indicated schematically in pink; its modulations are now known to have a $d$-symmetry form factor ${ }^{14-16}$. The range of hole density, $p$, in which $d$-symmetry form factor density waves are studied in this paper is indicated by the white double-headed arrow. b. Schematic of the electronic structure in a cuprate dFF-DW. Grey dots represent the Cu sites and correspond to the white dots in $\mathbf{c}$. The $\mathrm{O}_{x}$ and $\mathrm{O}_{y}$ sites within each $\mathrm{CuO}_{2}$ unit-cell are electronically inequivalent, as represented by a colour scale ranging from yellow through white to blue. The schematic DW modulates horizontally with wavelength $\lambda$, or with wavevector $\mathbf{Q}_{x}$ (horizontally), and with period $4 a_{0}$. The periodic modulations at $O_{x}$ sites are $\pi$ out of phase with those at $O_{y}$ sites, as seen by considering the two trajectories marked $\phi_{x}$ and $\phi_{y}$ (Supplementary Section 1). c, Measured $Z\left(\mathbf{r}, 150 \mathrm{meV}\right.$ ) at $T>T_{\mathbf{c}}$ in the pseudogap phase of $\mathrm{Bi}_{2} \mathrm{Sr}_{2} \mathrm{CaCu}_{2} \mathrm{O}_{8}$ at hole density $p \sim 8 \%$. Two periods of dFF-DW modulation at $\mathbf{Q}_{x}$ that correspond directly to the schematic in $\mathbf{b}$ are shown. Thus, to observe the dFF-DW state, sublattice-phase-resolved imaging is required and achieved here in the pseudogap regime. The transparent overlay shows the relationship between locations of $\mathrm{Cu}, \mathrm{O}_{x}, \mathrm{O}_{y}$ atoms in the $\mathrm{CuO}$ plane and the dFF-DW, whose wavevector here is along the $x$-direction

only the measured values of $Z(\mathbf{r})$ at $\mathrm{Cu}$ sites, whereas the other two, $O_{x}(\mathbf{r})$ and $O_{y}(\mathbf{r})$, contain only the measurements at the $x / y$-axis oxygen sites. Phase-resolved Fourier transforms of the $O_{x}(\mathbf{r})$ and $O_{y}(\mathbf{r})$ sublattice images ${ }^{14}, \tilde{O}_{x}(\mathbf{q})=\operatorname{Re} \tilde{O}_{x}(\mathbf{q})+i \operatorname{Im} \tilde{O}_{x}(\mathbf{q}) ;$ $\tilde{O}_{y}(\mathbf{q})=\operatorname{Re} \tilde{O}_{y}(\mathbf{q})+i \operatorname{Im} \tilde{O}_{y}(\mathbf{q})$, are used to determine the form factor symmetry for modulations at any $\mathbf{q}$

$$
\begin{gathered}
\tilde{D}^{Z}(\mathbf{q})=\frac{\left(\tilde{O}_{x}(\mathbf{q})-\tilde{O}_{y}(\mathbf{q})\right)}{2} \\
\tilde{S}^{\prime Z}(\mathbf{q})=\frac{\left(\tilde{O}_{x}(\mathbf{q})+\tilde{O}_{y}(\mathbf{q})\right)}{2} \\
\tilde{S}^{Z}(\mathbf{q})=\widetilde{\mathrm{Cu}}(\mathbf{q})
\end{gathered}
$$

where the superscript $Z$ identifies the type of sublattice-resolved data used. Specifically for a DW occurring at $\mathbf{Q}$, one can then evaluate the magnitude of its $d$-symmetry form factor $\tilde{D}(\mathbf{Q})$ and its $s^{\prime}$ - and $s$-symmetry form factors $\tilde{S}^{\prime}(\mathbf{Q})$ and $\tilde{S}(\mathbf{Q})$, respectively. Studies of non-energy-resolved $R(\mathbf{r}, E)$ images using this approach have revealed that the DW modulations in the $O_{x}(\mathbf{r})$ and $O_{y}(\mathbf{r})$ sublattice images of electronic structure in underdoped $\mathrm{Bi}_{2} \mathrm{Sr}_{2} \mathrm{CaCu}_{2} \mathrm{O}_{8+x}$ and $\mathrm{Ca}_{2-x} \mathrm{Na}_{x} \mathrm{CuO}_{2} \mathrm{Cl}_{2}$ consistently exhibit a relative phase of $\pi$ and therefore a predominant $d$-symmetry form factor $^{14}$; X-ray scattering studies ${ }^{15,16}$ yield the same conclusion for two other cuprates, $\mathrm{YBa}_{2} \mathrm{Cu}_{3} \mathrm{O}_{7-x}$ and $\mathrm{Bi}_{2} \mathrm{Sr}_{2-x} \mathrm{La}_{x} \mathrm{CuO}_{6+\delta}$.

Such $\mathrm{X}$-ray scattering studies now generally report a short-ranged density wave with wavevector centred around $\mathbf{Q}=(Q, 0) ;(0, Q)$ occurring approximately in the pink shaded regions ${ }^{11-13}$ of the schematic phase diagram in Fig. 1a. Figure 1b,c exemplifies the predominately $d$-symmetry form factor ${ }^{14-16}$ of this DW when imaged directly. One obstacle to understanding this dFF-DW state is that large-field-of-view sublattice-resolved images of cuprate electronic structure ${ }^{14}$ never look like an ideal long-range ordered version of Fig. 1b. Instead, Fig. 2a shows a typical $Z(\mathbf{r}$, $150 \mathrm{meV}$ ) image of $p=8 \% \mathrm{Bi}_{2} \mathrm{Sr}_{2} \mathrm{CaCu}_{2} \mathrm{O}_{8+x}$, for $T \ll T_{c}$ in the superconducting phase, whereas Fig. $2 \mathrm{~b}$ shows the equivalent $Z$ (r), $150 \mathrm{meV}$ ) for $T>T_{\mathrm{c}}$ in the cuprate pseudogap phase. Although elements indistinguishable from Fig. $1 c$ can be seen in $2 a, b$, no long-range order is obvious. Therefore, to explore the spatial arrangements of the dFF-DW in such electronic-structure images, we analyse $\tilde{D}^{Z}(\mathbf{q})$, which is a robust sublattice-phase-resolved measure of the $d$-symmetry form factor (Supplementary Section 3). Analysis of Fig. 2a,b in this fashion yields Fig. 2c,d; both clearly exhibit the dFF-DW maxima at the two inequivalent wavevectors $\mathbf{Q}_{x}$ and $\mathbf{Q}_{y}$. Fourier filtering these two $\tilde{D}^{Z}(\mathbf{q})$ from Fig. 2a,b for 
a

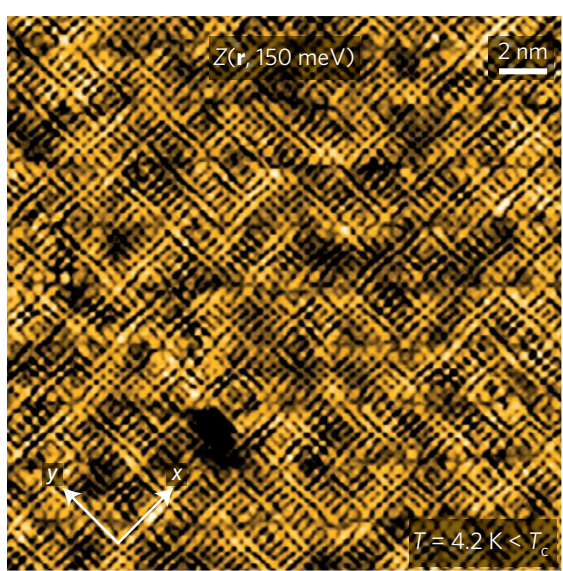

\section{b}

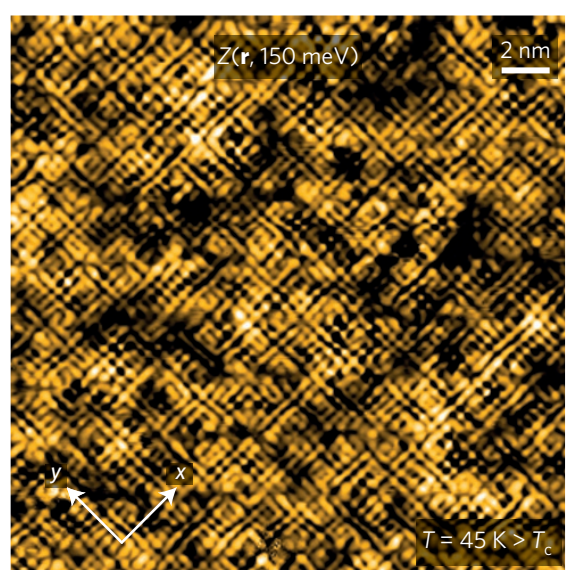

c

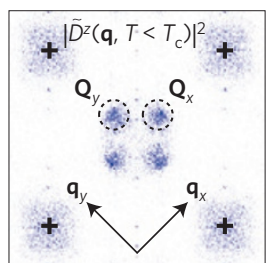

d

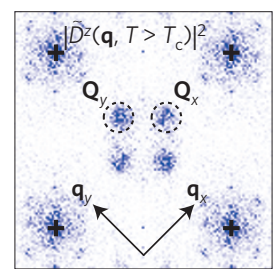

e

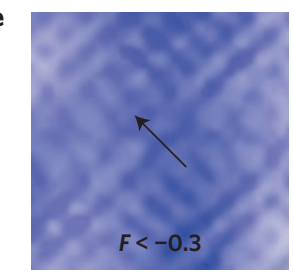

f

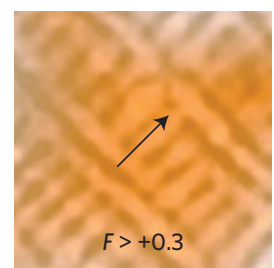

g

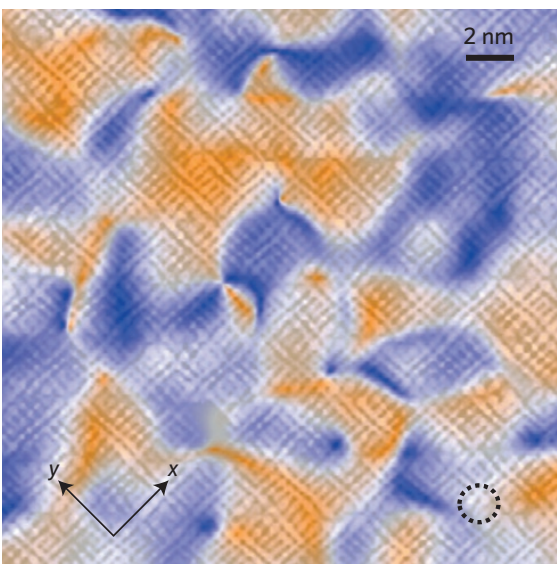

h

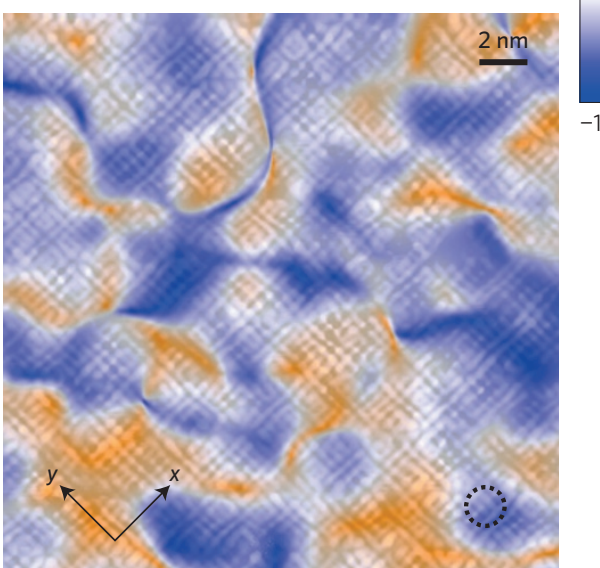

Figure 2 | dFF-DW domains in superconducting and pseudogap phases. a, Measured $Z\left(\mathbf{r}, 150\right.$ meV) at $T \ll T_{\mathrm{C}}$ in the superconducting phase of hole density $p \sim 8 \%$-doped $\mathrm{Bi}_{2} \mathrm{Sr}_{2} \mathrm{CaCu}_{2} \mathrm{O}_{8+x}(T \sim 4.2 \mathrm{~K})$. These complex spatial features involve modulations that comprehensively maintain a relative phase of $\pi$ between $O_{x}$ and $O_{y}$ in a disordered $d$-symmetry FF density wave. $\mathbf{b}$, Measured $Z$ ( $\mathbf{r}, 150$ meV) at $T>T_{c}$ in the pseudogap phase of hole density $p \sim 8 \%$-doped $\mathrm{Bi}_{2} \mathrm{Sr}_{2} \mathrm{CaCu}_{2} \mathrm{O}_{8}(T \sim 45 \mathrm{~K})$. Although correlation lengths are shorter, the dFF-DW phenomena are otherwise indistinguishable from observations at $T \ll T_{c}$. c. The $d$-symmetry form factor power spectral density $\left|\tilde{D}^{2}(\mathbf{q})\right|^{2}=\left|\left(\tilde{O}_{x}(\mathbf{q})-\tilde{O}_{y}(\mathbf{q})\right) / 2\right|^{2}$ determined from sublattice-phase-resolved analysis of data in $\mathbf{a}$. Two primary DW peaks at $\mathbf{Q}_{x}$ and $\mathbf{Q}_{y}$ exist with this $d$-symmetry form factor, as identified by dashed circles. $\mathbf{d}$, The $d$-symmetry FF power spectral density $\left|\tilde{D}^{Z}(\mathbf{q})\right|^{2}=\left|\left(\tilde{O}_{x}(\mathbf{q})-\tilde{O}_{y}(\mathbf{q})\right) / 2\right|^{2}$ determined from sublattice-phase-resolved analysis of data in $\mathbf{b}$. Again, two primary DW peaks at $\mathbf{Q}_{x}$ and $\mathbf{Q}_{y}$ exist with this $d$-symmetry form factor, showing that the $\mathbf{q}$-space structure of dFF-DW phenomenology is identical in the pseudogap phase and in the superconducting phase. e,f, Using only the regions within the dashed circles in $\mathbf{c}, \mathbf{d}$, the $\mathbf{r}$-space amplitudes of the dFF-DW in $\mathbf{a}, \mathbf{b}$ are calculated for modulations along $\mathbf{Q}_{x}$ from (5), and along $\mathbf{Q}_{y}$ from (6). Then using $F(\mathbf{r})=\left(\left|D_{x}(\mathbf{r})\right|-\left|D_{y}(\mathbf{r})\right|\right) /\left(\left|D_{x}(\mathbf{r})+\right| D_{y}(\mathbf{r}) \mid\right)($ see Supplementary Section 3$)$ regions primarily modulating along $y$-axis with $-1.0<F(\mathbf{r})<-0.3$ are shaded blue. Regions primarily modulating along $x$-axis with $+0.3<F(\mathbf{r})<+1.0$ are shaded orange. $\mathbf{g}$, Domain configuration of unidirectional dFF-DW modulations contained in Fig. 1a at $T \ll T_{\mathrm{C}}$. The unidirectionality colour scale for $F(\mathbf{r}$ ) demonstrated in $\mathbf{e}, \mathbf{f}$ is overlaid on the data in $\mathbf{a}$. The dashed circle shows the $\mathbf{r}$-space radius equivalent to the $\mathbf{q}$-space filter used to generate the $D_{x, y}(\mathbf{r})$ images by Fourier filtering (see Supplementary Section 4). h, Domain configuration of unidirectional dFF-DW modulations contained in Fig. $1 b$ at $T>T_{\mathrm{C}}$. The unidirectionality colour scale for $F(\mathbf{r})$ demonstrated in $\mathbf{e}, \mathbf{f}$ is overlaid on the data in $\mathbf{b}$. The dashed circle has same definition as in $\mathbf{g}$.

only those regions surrounding $\mathbf{Q}_{x}$ and $\mathbf{Q}_{y}$ (within dashed circles) generates two complex-valued $\mathbf{r}$-space images $D_{x}(\mathbf{r}), D_{y}(\mathbf{r})$

$$
\begin{aligned}
& D_{x}(\mathbf{r})=\frac{2}{(2 \pi)^{2}} \int \mathrm{d} \mathbf{q} \mathrm{e}^{i \mathbf{q} \cdot \mathbf{r}} \tilde{D}^{Z}(\mathbf{q}) \mathrm{e}^{-\frac{\left(\mathbf{q}-\mathbf{Q}_{x}\right)^{2}}{2 \Lambda^{2}}} \\
& D_{y}(\mathbf{r})=\frac{2}{(2 \pi)^{2}} \int \mathrm{d} \mathbf{q} \mathrm{e}^{i \mathbf{q} \cdot \mathbf{r}} \tilde{D}^{Z}(\mathbf{q}) \mathrm{e}^{-\frac{\left(\mathbf{q}-\mathbf{Q}_{y}\right)^{2}}{2 \Lambda^{2}}}
\end{aligned}
$$

where $\Lambda^{-1}$ is the characteristic length scale over which variations in $D_{x}(\mathbf{r}), D_{y}(\mathbf{r})$ can be resolved, and is set by the filter width in Fourier space.

Their magnitudes

$$
\begin{aligned}
& \left|D_{x}(\mathbf{r})\right|=\sqrt{\left(\operatorname{Re} D_{x}(\mathbf{r})\right)^{2}+\left(\operatorname{Im} D_{x}(\mathbf{r})\right)^{2}} \\
& \left|D_{y}(\mathbf{r})\right|=\sqrt{\left(\operatorname{Re} D_{y}(\mathbf{r})\right)^{2}+\left(\operatorname{Im} D_{y}(\mathbf{r})\right)^{2}}
\end{aligned}
$$

represent the local amplitudes of dFF-DW modulations along $\mathbf{Q}_{x}$ and $\mathbf{Q}_{y}$, respectively. Any unidirectional domain arrangements of the dFF-DW state can then be determined by introducing

$$
F(\mathbf{r})=\frac{\left|D_{x}(\mathbf{r})\right|-\left|D_{y}(\mathbf{r})\right|}{\left|D_{x}(\mathbf{r})\right|+\left|D_{y}(\mathbf{r})\right|}
$$

which is designed to identify regions where the dFF-DW modulation is primarily along the $x$-axis or the $y$-axis, depending on the sign of $F(\mathbf{r})$ (Supplementary Section 4). Figure 2e,f shows how regions of $-1.0<F(\mathbf{r})<-0.3$ (shaded blue) are primarily modulating along $y$-axis whereas regions $+0.3<F(\mathbf{r})<+1.0$ (shaded orange) are primarily modulating along $x$-axis (those with $-0.3<F(\mathbf{r})<+0.3$ shaded white appear at boundaries). Figure $2 \mathrm{~g}, \mathrm{~h}$ reveals the results of this analysis for the data in Fig. 2a,b respectively. Overall, the system is configured into spatial regions within which the dFF-DW along only one direction 
a

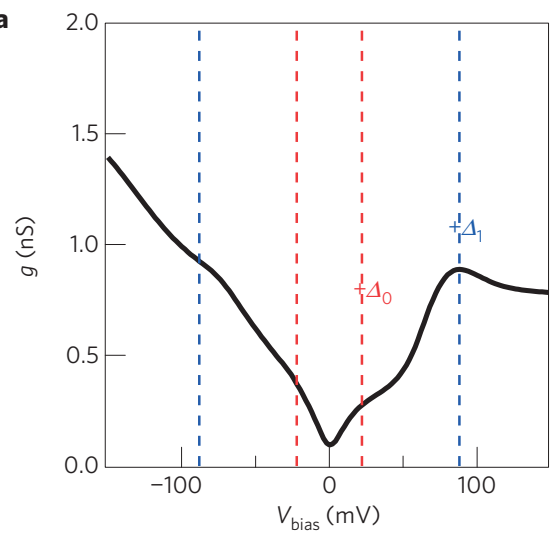

d

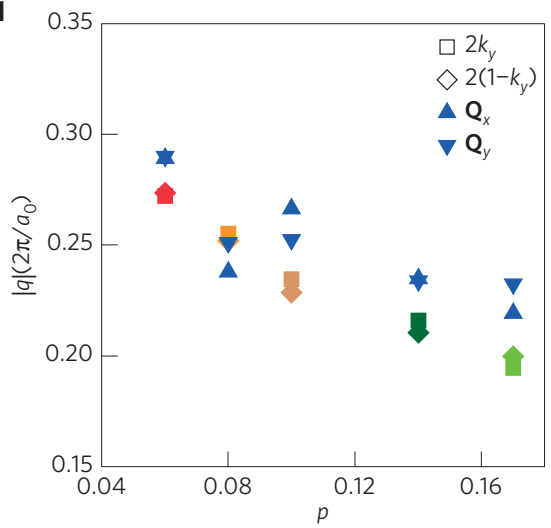

b

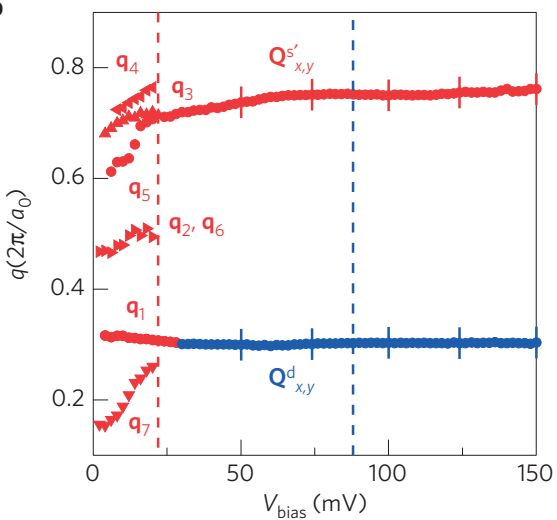

e

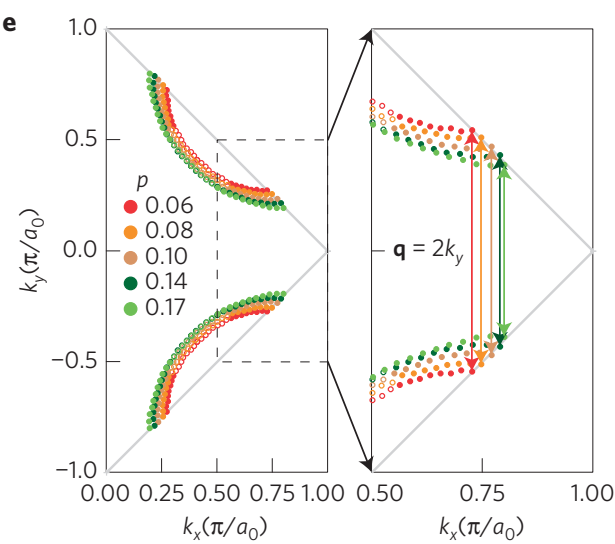

c

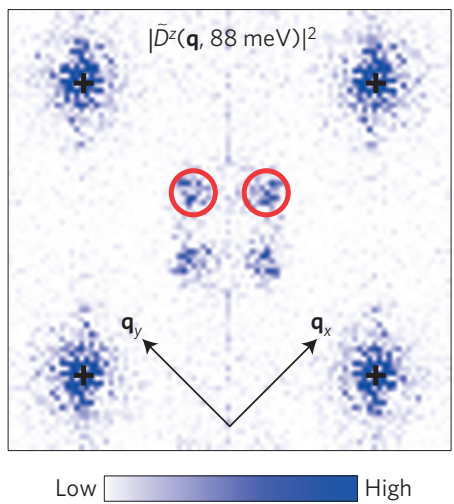

f

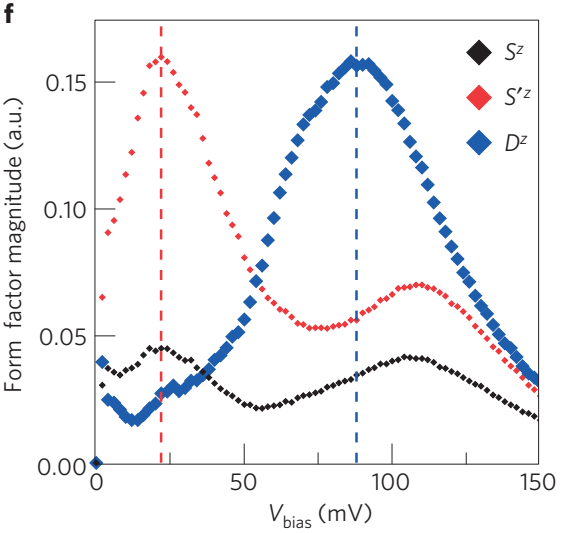

Figure 3 | Concentration of dFF-DW spectral weight on pseudogap energy. $\mathbf{a}$, The tunnelling density of states spectrum $g(E=\mathrm{eV})=\mathrm{d} l / \mathrm{d} V(E)$ typical of underdoped cuprates shown for the $p \sim 6 \%$ samples presented in this paper. The energy $\Delta_{0}$ beyond which Bogoliubov QPI does not exist ${ }^{31,32}$ and the pseudogap energy $\Delta_{1}$ are indicated. $\mathbf{b}$. The energy dispersion of seven dispersive modulations characteristic of Bogoliubov quasiparticle excitations of a $d$-wave superconductor $\left(\mathbf{q}_{1} \ldots \mathbf{q}_{7}\right)$ shown as red triangles, except for $\mathbf{q}_{1}$ and $\mathbf{q}_{5}$ which are plotted as red circles. These Bogoliubov quasiparticle interference modulations are all simultaneously observable only below the energy $\Delta_{0}$, as indicated by dashed red line (refs $30-32$ ); here we demonstrate that they exhibit a predominantly s'-symmetry form factor, indicated by the red colour. At energies above $\Delta_{0}$, the electronic-structure images evolve quickly to consist of only non-dispersive $\mathbf{Q}_{x}{ }^{d}$ and $\mathbf{Q}_{y}{ }^{d}$ wavevectors of the $d$-symmetry form factor DW. We plot the dispersion of these modulations by showing the energy dependence of the maxima in $\tilde{D}(\mathbf{q}, E)$ as blue squares, with the blue bars representing the one $\sigma$ width of each such peak. The same physical modulations when analysed using $\widetilde{S^{\prime}}(\mathbf{q}, E)$ appear as the non-dispersive $\mathbf{Q}_{x}{ }^{{ }^{\prime}}$ and $\mathbf{Q}_{y}{ }^{s^{\prime}}$ wavevectors shown as red circles, with the red bars representing the one $\sigma$ width of each such peak. c. Measured $\left|\tilde{D}^{Z}(\mathbf{q}, 88 \mathrm{meV})\right|^{2}$ for samples studied herein. The $\mathbf{Q}_{x}{ }^{d}$ and $\mathbf{Q}_{y}{ }^{d}$ wavevectors of the $d$-symmetry form factor DW are indicated by two red circles; the data of relevance for determining the energy/momentum dependence of the dFF-DW modulations is contained within each circle. $\mathbf{d}$, Measured doping dependence of the conventionally defined $\mathbf{Q}_{x}$ and $\mathbf{Q}_{y}$ of the $d$-symmetry form factor DW in underdoped $\mathrm{Bi}_{2} \mathrm{Sr}_{2} \mathrm{CaCu}_{2} \mathrm{O}_{8+x}$ is shown using blue symbols. The measured doping dependence of the $\mathbf{q}$-vector linking tips of arcs beyond which the signature of Bogoliubov quasiparticles disappears (e and refs 30-32) is shown by all other colours. e, Measured doping dependence of the wavevectors interconnecting the $\mathbf{k}$-space arc tips at which Bogoliubov quasiparticle signatures disappear ( $\mathbf{b}$ and refs 30-32). $\mathbf{f}$, Measured energy dependence of $S^{\prime}(E)$, where $\mathbf{q}$ is integrated over the region inside the solid red circles in $\mathbf{c}$, is shown in red. Measured energy dependence of $S(E)$, where $\mathbf{q}$ is integrated over the region inside the solid red circles in $\mathbf{c}$, is shown in black. Measured energy dependence of the $d$-symmetry form factor $D(E)$, where $\mathbf{q}$ is integrated over the region inside the solid red circles in c, is shown in blue (Supplementary Section 4). These data reveal that the dFF-DW spectral weight is concentrated at energies surrounding $\sim 90 \mathrm{meV}$, which, at this hole density, is the independently measured pseudogap energy scale $\Delta_{1}$ (see $\mathbf{a}$ ), and is indicated on all relevant panels by a dashed blue line.

is dominant. By overlaying the colour scale for $F(\mathbf{r})$ on the data in Fig. 2a,b to create Fig. 2g,h, one can see directly the unidirectional region configurations derived from equation (7). These observations of coexisting nanoscale unidirectional regions are in reasonable agreement with deductions from related $\mathrm{X}$-ray studies ${ }^{36}$ of $\mathrm{YBa}_{2} \mathrm{Cu}_{3} \mathrm{O}_{7-x}$. Finally, because the data in Fig. $2 \mathrm{~b}$ and Fig. 2h were measured at $T>T_{c}$ (pink region Fig. 1a), this demonstrates directly that the cuprate dFF-DW appears first in the non-superconducting 'pseudogap' regime.

A conventional CDW state opens a gap in the energy spectrum of $\mathbf{k}$-space electronic eigenstates with the maximum spectral weight of modulating states occurring at the edges of this energy gap ${ }^{33}$. But which energy gap (if any) is associated with the dFF-DW state found in underdoped cuprates is unknown. Figure 3a shows how a typical tunnelling conductance spectrum representative of strongly underdoped cuprates exhibits two characteristic energies ${ }^{30-32}$. Whereas the lower-energy scale $\Delta_{0}$ represents the maximum energy at which Bogoliubov quasiparticle excitations exist ${ }^{30-32}$ (see Fig. 3b), the higher-energy scale (dashed blue line) is the cuprate 'pseudogap' as determined from its comparison with the doping dependence of the pseudogap scale in tunnelling and photoemission. To identify the energy dependence of the cuprate dFF-DW states, we measure $Z(\mathbf{r},|E|)$, and from it calculate $\tilde{D}^{Z}(\mathbf{q},|E|), \tilde{S}^{Z}(\mathbf{q},|E|)$ and $\tilde{S}^{Z}(\mathbf{q},|E|)$. Figure $3 c$ shows the measured power spectral density of the $d$-symmetry FF modulations $\tilde{D}^{Z}(\mathbf{q},|88 \mathrm{meV}|)^{2}$, with the wavevectors near $\mathbf{Q}_{x}$ and $\mathbf{Q}_{y}$ indicated by red circles. Adopting the common convention in X-ray studies ${ }^{9-11,16}$ for estimating the DW wavevector magnitude $|\mathbf{Q}|$, we measure $\tilde{D}^{Z}(\mathbf{q},|E|)$ along a line 


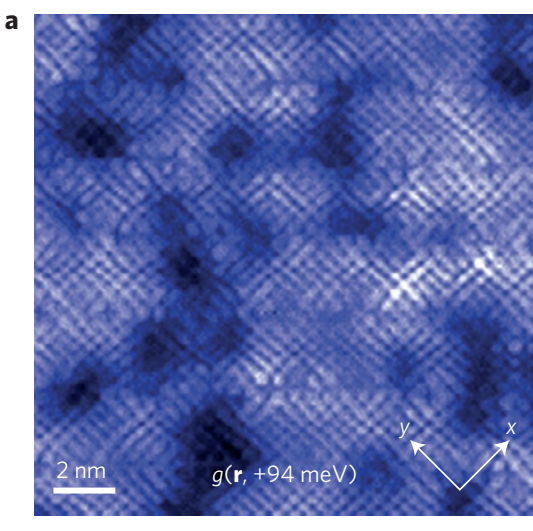

Low

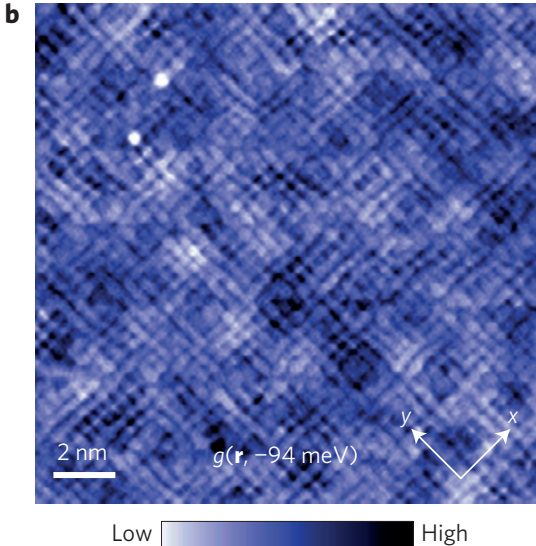

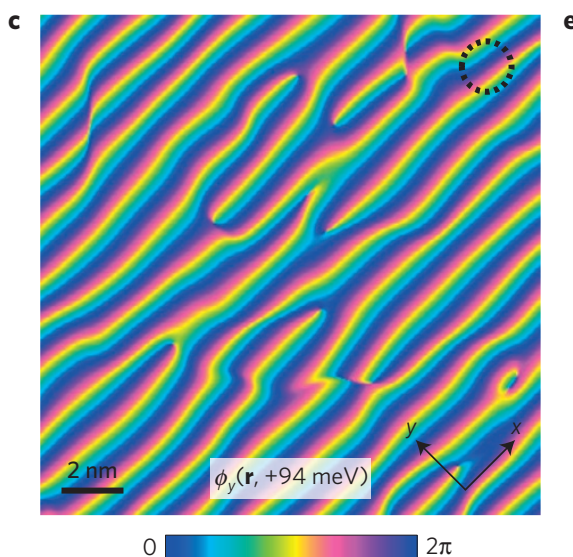

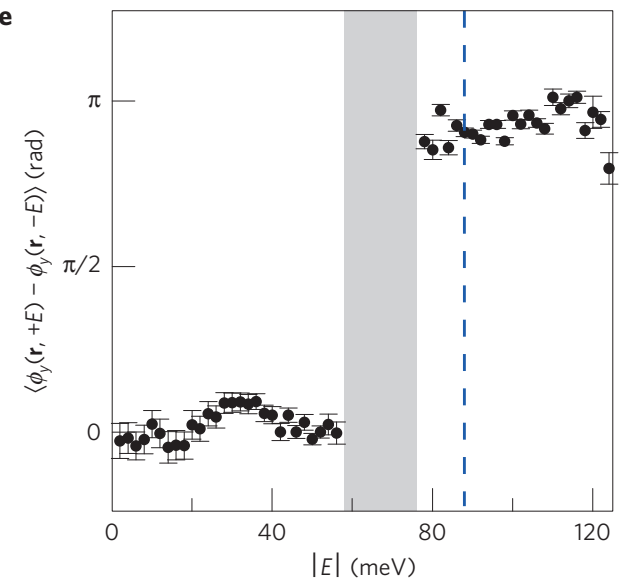

d
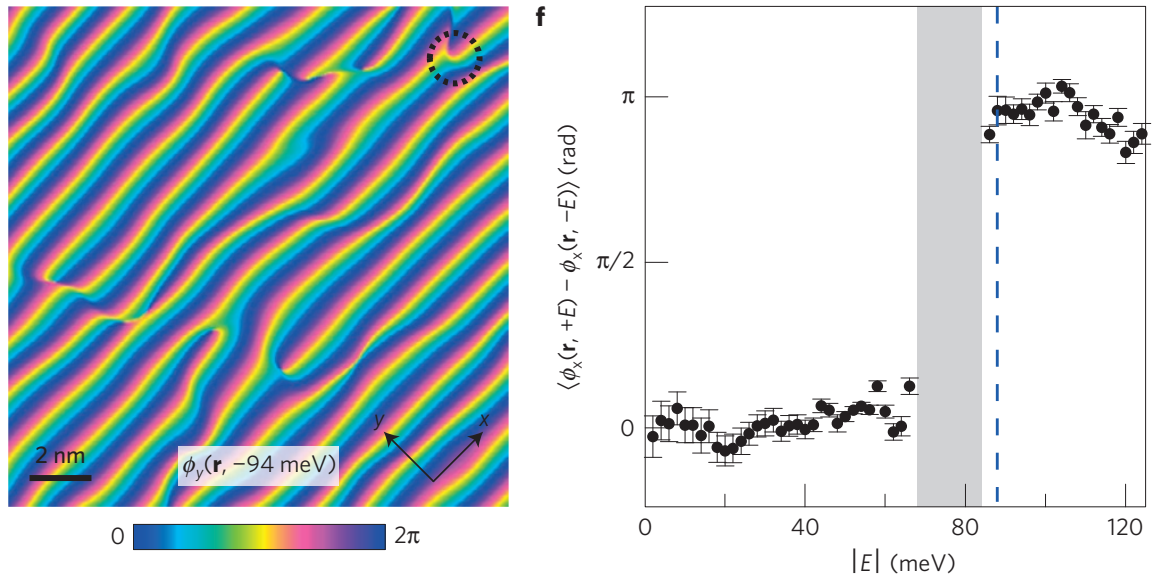

Figure 4 | Relationship between dFF-DW modulations of filled and empty states. a, Differential tunnelling conductance image $g(\mathbf{r},+94$ meV) measured above $E_{\mathrm{F}}$ near the pseudogap energy $+\Delta_{1}$. The colour scale is reversed compared to $\mathbf{b}$. $\mathbf{b}$, Differential tunnelling conductance image $g(\mathbf{r},-94$ meV)

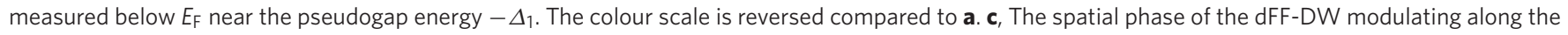
$y$-direction $\phi_{y}(\mathbf{r}, E)$ is calculated using equation (8) from $g(\mathbf{r},+94 \mathrm{meV})$ data in $\mathbf{a}$. The dashed circle shows the $\mathbf{r}$-space radius equivalent to the $\mathbf{q}$-space filter used to generate the $\phi_{x, y}(\mathbf{r}, E)$ images by Fourier filtering (see Supplementary Section 6). d, The spatial phase of the dFF-DW modulating along the

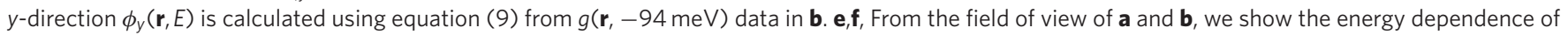

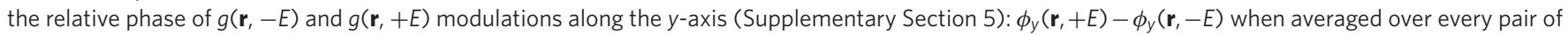

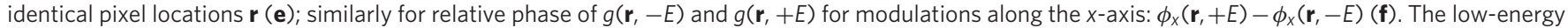
modulations at $+E$ and $-E$ are in phase spatially and so have a relative phase difference of 0 . As the pseudogap energy $\Delta_{1}$ is approached and the $d F F-D W$ phenomena emerge, the relative spatial phase of empty state and filled state dFF-DW modulations varies wildly in the narrow energy grange shaded grey, and the quickly develops a robust phase shift of $\pi$.

in the high-symmetry directions $(1,0):(0,1)$ passing through the region of the dFF-DW peak and fit these data to a background plus Gaussian; the peak positions of the two Gaussians are then assigned to be the values of $\mathbf{Q}_{x}$ and $\mathbf{Q}_{y}$. It is important to appreciate, however, that a wide range of $\mathbf{Q}$ values can actually be detected under each peak in $\tilde{D}^{Z}(\mathbf{q},|E|)$ and in X-ray scattering intensities; it remains to be determined whether these broad peaks with incommensurate maxima are due to domains of continuously incommensurate dFF-DW or domains of commensurate dFF-DW separated by discommensuration ${ }^{37}$. Nevertheless, Fig. 3b plots the energy dependence of the dFF-DW wavevectors (blue line) determined in this way for a $p=0.06$ sample. Such information was not previously available from measurements of the modulation wavevectors from STM images lacking sublattice-phase-resolved segregation $^{14}$ into $\tilde{O}_{x}(\mathbf{q})$ and $\tilde{O}_{y}(\mathbf{q})$. Figure $3 \mathrm{e}$ shows the measured k-space locus where Bogoliubov quasiparticles exist ${ }^{30-32}$ as a function of hole density. When the dispersive 'octet' of Bogoliubov scattering interference disappears, a transition occurs to an ultraslow dispersing density wave modulation (Fig. 3b). In Fig. 3d, the doping dependence of the conventional $\mathbf{Q}_{x}, \mathbf{Q}_{y}$ of the $d$-symmetry form factor modulations is shown using blue symbols, while the shortest wavevectors interconnecting the measured $\mathbf{k}$-space regions where Bogoliubov scattering interference disappears (Fig. 3e) are indicated by using coloured symbols referring to each hole density in Fig. 3e. These data demonstrate directly that the conventionally determined $\mathbf{Q}_{x}, \mathbf{Q}_{y}$ of the dFF-DW state correspond to the locations in k-space of these arc tips. Finally, in Fig. 3f we show the measured energy dependences of the amplitudes of the $s^{-}, s^{\prime}$ - and $d$-form factor modulations, $S^{Z}(E), S^{Z}(E)$ and $D^{Z}(E)$, determined by integrating over the region of $\mathbf{q}$-space enclosed by solid red circles in Fig. 3c (Supplementary Section 5). The $d$-symmetry form factor is negligible for modulations in the low-energy range that contains only Bogoliubov quasiparticles (and which we now see is dominated by $s^{\prime}$-symmetry form factors), but it rapidly becomes intense at higher energy and reaches maximum at the pseudogap energy scale, which for this sample is $\Delta_{1} \sim 90 \mathrm{meV}$. This reveals that the characteristic energy of electronic-structure modulations in the cuprate $d$-symmetry FF density wave is actually the pseudogap energy.

As a function of energy, the transition from Bogoliubov quasiparticle interference (QPI) modulations to dFF-DW modulations occurs in an unusual fashion. Although Bogoliubov QPI is 
observed as expected everywhere on the Fermi surface in overdoped cuprates $^{32}$, in underdoped samples it evolves as expected only until the energy $E \approx \Delta_{0}$, at which the terminations of the Bogoliubov coherent k-space arcs (Fig. 3e) are observed ${ }^{30-32}$. Here, the set of seven dispersive scattering interference modulations $\mathbf{q}_{1}, \mathbf{q}_{2}, \ldots \mathbf{q}_{7}$ signifying Bogoliubons ${ }^{31}$ (Supplementary Section 5) disappears in a narrow energy window during which dispersion of the two surviving modulations $q_{1}(E)$ and $q_{5}(E)$ comes to a halt, leaving the ultra-slow dispersing dFF-DW modulations with $\mathbf{q}_{1}^{*} \approx \mathbf{q}_{1}\left(\Delta_{0}\right)$ and $\mathbf{q}_{5}^{*} \approx \mathbf{q}_{5}\left(\Delta_{0}\right)$ (see Fig. $3 \mathrm{~b}$ and refs $30-32$ ). The intensity of these non-dispersive modulations first becomes detectable at $\Delta_{0}$ and, as we show below, reaches an intense maximum at $\Delta_{1}$, all the while maintaining the same wavevectors $\mathbf{Q}_{x}{ }^{d}$ and $\mathbf{Q}_{y}{ }^{d}$, as shown in Fig. $3 \mathrm{~b}$. We refer to this $\mathbf{k}$-space region where Bogoliubov quasiparticles yield to modulations of a dFF-DW as the 'hot frontier' (we thank S. A. Kivelson for proposing the term 'hot frontier' to describe the k-space phenomenology of the cuprate dFF-DW as observed using spectroscopic imaging STM) to distinguish it from the colloquial 'hot spots' beyond which, in a conventional density wave, dispersive quasiparticle states would reappear. In cuprates, this does not occur and, instead, the 'hot frontiers' define the k-space limit beyond which only dFF-DW modulations are detected ${ }^{30-32}$ using spectroscopic imaging STM (blue in Fig. 3b).

Key information on the microscopic cause of any DW state is also contained in the relationship between modulations of states above and below the Fermi energy. For example, mixing via interactions of states with momenta $\mathbf{k}_{1}$ and $\mathbf{k}_{2}$ generates modulations at wavevector $\mathbf{Q}=\mathbf{k}_{1}-\mathbf{k}_{2}$. The wavefunctions of any resulting DW would then form bonding/anti-bonding states below/above the Fermi level which are proportional to $\mathrm{e}^{i \mathbf{k}_{1} \cdot \mathbf{r}} \pm \mathrm{e}^{i \mathbf{k}_{2} \cdot \mathbf{r}}$. The related densities of these states would then exhibit modulations governed by $\left|\mathrm{e}^{i \mathbf{k}_{1} \cdot \mathbf{r}} \pm \mathrm{e}^{i \mathbf{k}_{2} \cdot \mathbf{r}}\right|^{2}=2(1 \pm \cos (\mathbf{Q} \cdot \mathbf{r}))$. In such scenarios, the DW modulations above the Fermi energy should always be $\pi$ out of phase with the equivalent ones below. To explore this issue in $\mathrm{Bi}_{2} \mathrm{Sr}_{2} \mathrm{CaCu}_{2} \mathrm{O}_{8+x}$, we show in Fig. 4a,b the measured $g(\mathbf{r},+94 \mathrm{meV})$ from filled states and $g(\mathbf{r},-94 \mathrm{meV})$ from empty states, respectively, each at the characteristic energy of the dFF-DW (Fig. 3f and Supplementary Section 6); avoidance of the set-point error is discussed in Supplementary Section 6. For these two images the sublattice-phase-resolved $\tilde{D}^{g}(\mathbf{q}, E)$ equation (1) are calculated and reveal a predominantly $d$-symmetry form factor modulation with wavevectors near $\mathbf{Q}_{x}$ and $\mathbf{Q}_{y}$ in Fig. 4a,b. Next, by Fourier filtering these two $\tilde{D}^{g}(\mathbf{q}, E)$ for regions surrounding $\mathbf{Q}_{y}$, we determine the complex-valued $D_{y}(\mathbf{r})$, and thus the spatial phase of dFF-DW modulation along $\mathbf{Q}_{y}$ as

$$
\phi_{y}(\mathbf{r}, E)=\arctan \left(\operatorname{Im} D_{y}(\mathbf{r}, E) / \operatorname{Re} D_{y}(\mathbf{r}, E)\right)
$$

This is shown for $E=+94 \mathrm{meV}$ in Fig. $4 \mathrm{c}$ and for $E=-94 \mathrm{meV}$ in Fig. $4 \mathrm{~d}$. Visual comparison reveals that these two $\phi_{y}(\mathbf{r}, \pm E)$ images are out of phase with each other by $\pi$. And, indeed, the spatial-average value of $\phi_{y}(\mathbf{r},+E)-\phi_{y}(\mathbf{r},-E)$ as a function of $E$ (over the whole field of view of $\mathrm{A}$ and $\mathrm{B}$ ) is shown in Fig. 4e. It reveals that, whereas the relevant $\mathbf{Q}_{x}$ and $\mathbf{Q}_{y}$ components of $g(\mathbf{r},+E)$ and $g(\mathbf{r},-E)$ images are in phase with each other at low energy, they rapidly evolve at $|E|>70 \mathrm{meV}$ and become globally $\pi$ out of phase at $|E| \sim \Delta_{1}$ (Fig. $\left.4 \mathrm{a}, \mathrm{b}\right)$. The shaded region indicates evolution through a situation where some areas exhibit $\phi \sim 0$ and some $\phi \sim \pi$, but this is quickly resolved on reaching the pseudogap energy $\Delta_{1}$. Similar analysis for the particle-hole symmetry in phases $\phi_{x}(\mathbf{r}, \pm E)$ of $\mathbf{Q}_{x}$ modulations

$$
\phi_{x}(\mathbf{r}, E)=\arctan \left(\operatorname{Im} D_{x}(\mathbf{r}, E) / \operatorname{Re} D_{x}(\mathbf{r}, E)\right)
$$

yields a virtually identical result (Fig. 4f). These phenomena also occur throughout the underdoped regions of the phase diagram
(Supplementary Section 6.III), demonstrating that, in the cuprate dFF-DW state, a phase difference of $\pi$ exists between spatial modulations of the filled states below energy $E \sim-\Delta_{1}$ and the empty states above $E \sim+\Delta_{1}$.

To summarize: by introducing techniques to determine the energy/momentum and doping dependence of modulation form factor symmetry, we find that the predominantly $d$-symmetry form factor density wave exists throughout the underdoped region of the $\mathrm{Bi}_{2} \mathrm{Sr}_{2} \mathrm{CaCu}_{2} \mathrm{O}_{8}$ phase diagram (Fig. 3d), including in the pseudogap regime $T>T_{c}$ (Figs $1 \mathrm{c}$ and $2 \mathrm{~b}$ ). The spatial arrangements are primarily in the form of nanoscale regions, each containing a primarily unidirectional dFF-DW (Fig. 2g,h). The conventionally defined wavevectors $\mathbf{Q}_{x}$ and $\mathbf{Q}_{y}$ of the dFF-DW state evolve with doping as determined by the four shortest scattering vectors linking the $\mathbf{k}$-space regions beyond which Bogoliubov quasiparticle excitations are non-existent (Fig. 3d,e) and at which the pseudogap emerges. Further, we demonstrate that, as determined in terms of tunnelling probabilities, the dFF-DW state is particle-hole antisymmetric, in the sense that a phase difference of $\pi$ exists between spatial modulations of the filled states $\left(E \sim-\Delta_{1}\right)$ and the empty states $\left(E \sim+\Delta_{1}\right)$ (Fig. $\left.4 \mathrm{e}, \mathrm{f}\right)$. Perhaps most significantly, we show that the characteristic energy of the cuprate dFF-DW state is actually the pseudogap energy $\Delta_{1}$ (Fig. 3f).

These data provide clear evidence that the cuprate $d$-symmetry form factor density wave state involves particle-hole interactions, and that these occur primarily very near wavevectors interconnecting the 'hot frontiers' in $\mathbf{k}$-space at which the pseudogap emerges $^{30-32}$. Moreover, the dFF-DW electronic-structure modulations have a characteristic energy scale which is the pseudogap energy. This intimate connection of the dFF-DW state with the pseudogap electronic structure is consistent with the fact that this state is found only within the pseudogap regime ${ }^{11-13}$. Of course, electronlattice interactions can also play a significant role, with the coupling to the $B_{1 g}$ modes long being of foremost interest ${ }^{19,20,38}$. Strong interactions of this mode with the electrons ${ }^{39}$ ultimately leading to static, finite-Q lattice distortions with $d$-symmetry form factor ${ }^{15}$ have recently been discovered in association with the cuprate dFFDW state. Nevertheless, electron-lattice interactions are not by themselves sufficient to explain the phase diagram of the dFF-DW (refs 11-13) because, for example, they also exist in the overdoped regime where the dFF-DW is absent. Moreover, theoretical models involving $\mathbf{k}$-space instabilities ${ }^{26,27,29,40}$ which are consistent with the results herein, emphasize that a density wave with this $\mathbf{Q}$ and form factor symmetry cannot emerge from a large Fermi surface; instead, a pre-existing reorganization of $\mathbf{k}$-space due to the pseudogap would be required. Overall, our data support a microscopic picture in which the exotic electronic structure of the pseudogap is parent to the dFF-DW state and not vice versa, where the energy scale and wavevectors of the dFF-DW are intimately linked to those of the pseudogap, and in which the $d$-symmetry DW competes directly for spectral weight with the $d$-symmetry superconductor at the $\mathbf{k}$-space 'hot frontier' between superconductivity and the pseudogap.

Received 18 May 2015; accepted 16 September 2015; published online 26 October 2015

\section{References}

1. Orenstein, J. \& Millis, A. J. Advances in the physics of high-temperature superconductivity. Science 288, 468-474 (2000).

2. Norman, M. R., Pines, D. \& Kallin, C. The pseudogap: Friend or foe of high $T_{\mathrm{c}}$ ? Adv. Phys. 54, 715-733 (2005).

3. Wu, T. et al. Magnetic-field-induced charge-stripe order in the high-temperature superconductor $\mathrm{YBa}_{2} \mathrm{Cu}_{3} \mathrm{Oy}$. Nature 477, 191-194 (2011).

4. Chang, J. et al. Direct observation of competition between superconductivity and charge density wave order in $\mathrm{YBa}_{2} \mathrm{Cu}_{3} \mathrm{O}_{6.67}$. Nature Phys. 8, 871-876 (2012).

5. Ghiringhelli, G. et al. Long-range incommensurate charge fluctuations in (Y,Nd) $\mathrm{Ba}_{2} \mathrm{Cu}_{3} \mathrm{O}_{6+x}$. Science 337, 821-825 (2012). 
6. Achkar, A. J. et al. Distinct charge orders in the planes and chains of ortho-III-ordered $\mathrm{YBa}_{2} \mathrm{Cu}_{3} \mathrm{O}_{6+\delta}$ superconductors identified by resonant elastic X-ray scattering. Phys. Rev. Lett. 109, 167001 (2012).

7. Torchinsky, D. H., Mahmood, F., Bollinger, A. T., Božović, I. \& Gedik, N. Fluctuating charge-density waves in a cuprate superconductor. Nature Mater. 12, 387-391 (2013)

8. Blackburn, E. et al. X-ray diffraction observations of a charge-density-wave order in superconducting ortho-II $\mathrm{YBa}_{2} \mathrm{Cu}_{3} \mathrm{O}_{6.54}$ single crystals in zero magnetic field. Phys. Rev. Lett. 110, 137004 (2013)

9. Comin, R. et al. Charge order driven by Fermi-arc instability in $\mathrm{Bi}_{2} \mathrm{Sr}_{2-x} \mathrm{La}_{x} \mathrm{CuO}_{6+\delta}$. Science 343, 390-392 (2014).

10. da Silva Neto, E. H. et al. Ubiquitous interplay between charge ordering and high-temperature superconductivity in cuprates. Science 343, 393-396 (2014)

11. Blanco-Canosa, S. et al. Resonant X-ray scattering study of charge-density wave correlations in $\mathrm{YBa}_{2} \mathrm{Cu}_{3} \mathrm{O}_{6+x}$. Phys. Rev. B 90, 054513 (2014).

12. Hücker, M. et al. Competing charge, spin, and superconducting orders in underdoped $\mathrm{YBa}_{2} \mathrm{Cu}_{3} \mathrm{O}_{y}$. Phys. Rev. B 90, 054514 (2014).

13. Blackburn, E. X-ray diffraction studies of charge density waves in cuprate superconductors: A brief review. Physica B 460, 132-135 (2014).

14. Fujita, K. et al. Direct phase-sensitive identification of a $d$-form factor density wave in underdoped cuprates. Proc. Natl Acad. Sci. USA 111, E3026-E3032 (2014)

15. Forgan, E. M. et al. The nature of the charge density waves in under-doped $\mathrm{YBa}_{2} \mathrm{Cu}_{3} \mathrm{O}_{6.54}$ revealed by X-ray measurements of the ionic displacements. Preprint at http://arXiv.org/abs/1504.01585 (2015)

16. Comin, R. et al. The symmetry of charge order in cuprates. Nature Mater. 14, 796-800 (2015)

17. Li, J.-X., Wu, C.-Q. \& Lee, D.-H. Checkerboard charge density wave and pseudogap of high- $T_{c}$ cuprate. Phys. Rev. B 74, 184515 (2006).

18. Seo, K., Chen, H.-D. \& Hu, J. d-wave checkerboard order in cuprates. Phys. Rev. B 76, 020511 (2007).

19. Fu, H. C., Honerkampand, C. \& Lee, D.-H. Renormalization group study of the electron-phonon interaction in high- $\mathrm{T}_{\mathrm{c}}$ cuprates. Europhys. Lett. 75, 146-152 (2006)

20. Newns, D. M. \& Tsuei, C. C. Fluctuating Cu-O-Cu bond model of high-temperature superconductivity. Nature Phys. 3, 184-191 (2007)

21. Sachdev, S. \& La Placa, R. Bond order in two-dimensional metals with antiferromagnetic exchange interactions. Phys. Rev. Lett. 111, 027202 (2013).

22. Davis, J. C. \& Lee, D.-H. Concepts relating magnetic interactions, intertwine electronic orders, and strongly correlated superconductivity. Proc. Natl Acad. Sci. USA 110, 17623-17630 (2013).

23. Allais, A., Bauer, J. \& Sachdev, S. Density wave instabilities in a correlated two-dimensional metal. Phys. Rev. B 90, 155114 (2014).

24. Fischer, M. H., Wu, S., Lawler, M., Paramekanti, A. \& Kim, E.-A. Nematic and spin-charge orders driven by hole-doping a charge-transfer insulator. New J. Phys. 16, 093057 (2014)

25. Pépin, C., de Carvahlo, V. S., Kloss, T. \& Montiel, X. Coexistence of pseudo-gap and charge order at the hot spots in cuprate superconductors. Phys. Rev. B 90, 195207 (2014).

26. Chowdhury, D. \& Sachdev, S. Density wave instabilities of fractionalized Fermi liquids. Phys. Rev. B 90, 245136 (2014).

27. Atkinson, W. A., Kampf, A. \& Bulut, S. Charge order in the pseudogap phase of cuprate superconductors. New J. Phys. 17, 013025 (2015)

28. Yamakawa, Y. \& Kontani, H. Spin-fluctuation-driven nematic charge-density-wave in cuprate superconductors: Charge-orbital-spin multimode fluctuations caused by vertex corrections. Phys. Rev. Lett. 114, 257001 (2015).

29. Zhang, L. \& Mei, J.-W. Reconstructed Fermi surface and quantum oscillation of doped resonating valence bond state with incommensurate charge order in underdoped cuprates. Preprint at http://arXiv.org/abs/1408.6592 (2014).
30. Kohsaka, Y. et al. How Cooper pairs vanish approaching the Mott insulator in $\mathrm{Bi}_{2} \mathrm{Sr}_{2} \mathrm{CaCu}_{2} \mathrm{O}_{8+\delta}$. Nature 454, 1072-1078 (2008).

31. Fujita, K. et al. Spectroscopic imaging scanning tunneling microscopy studies of electronic structure in the superconducting and pseudogap phases of cuprate high- $\mathrm{T}_{c}$ superconductors. J. Phys. Soc. Jpn 81, 011005 (2011).

32. Fujita, K. et al. Simultaneous transitions in cuprate momentum-space topology and electronic symmetry breaking. Science 344, 612-616 (2014)

33. Grüner, G. Density Waves in Solids 31-70 (Westview Press, 1994)

34. Nayak, C. Density-wave states of nonzero angular momentum. Phys. Rev. B 62, 4880-4889 (2000)

35. Metlitski, M. A. \& Sachdev, S. Quantum phase transitions of metals in two spatial dimensions. I. Ising-nematic order. Phys. Rev. B 82, 075127 (2010).

36. Comin, R. et al. Broken translational and rotational symmetry via charge stripe order in underdoped $\mathrm{YBa}_{2} \mathrm{Cu}_{3} \mathrm{O}_{6+y}$. Science 347, 1335-1339 (2015).

37. Burk, B. et al. Charge-density-wave domains in $1 T-\mathrm{TaS}_{2}$ observed by satellite structure in scanning-tunneling-microscopy images. Phys. Rev. Lett. 66, 3040-3043 (1991)

38. Mishra, V. \& Norman, M. A strong-coupling critique of spin fluctuation driven charge order in undoped cuprates. Phys. Rev. B 92, 060507(R) (2015).

39. Le Tacon, M. et al. Inelastic X-ray scattering in $\mathrm{YBa}_{2} \mathrm{Cu}_{3} \mathrm{O}_{6.6}$ reveals giant phonon anomalies and elastic central peak due to charge-density-wave formation. Nature Phys. 10, 52-58 (2014).

40. Kloss, T. et al. SU(2) symmetry in a realistic spin-fermion model for cuprate superconductors. Phys. Rev. B 91, 205124 (2015).

\section{Acknowledgements}

We acknowledge and thank H. Alloul, D. Chowdhury, R. Comin, A. Damascelli, E. Fradkin, D. Hawthorn, S. Hayden, J. E. Hoffman, M.-H. Julien, D. H. Lee, M. Norman and C. Pepin for helpful discussions and communications. We are especially grateful to S. A. Kivelson for key scientific discussions and advice. Experimental studies were supported by the Center for Emergent Superconductivity, an Energy Frontier Research Center, headquartered at Brookhaven National Laboratory and funded by the US Department of Energy under DE-2009-BNL-PM015, as well as by a Grant-in-Aid for Scientific Research from the Ministry of Science and Education (Japan) and the Global Centers of Excellence Program for Japan Society for the Promotion of Science. C.K.K. acknowledges support under the FlucTeam Program at Brookhaven National Laboratory (Contract DE-AC02-98CH10886). S.D.E., J.C.D. and A.P.M. acknowledge the support of EPSRC through the Programme Grant 'Topological Protection and Non-Equilibrium States in Correlated Electron Systems'. Theoretical studies at Cornell University were supported by the US Department of Energy, Office of Basic Energy Sciences, Division of Materials Science and Engineering under Award DE-SC0010313. Theoretical studies at Harvard University were supported by NSF Grant DMR-1103860 and by the Templeton Foundation. Research at Perimeter Institute is supported by the Government of Canada through Industry Canada and by the Province of Ontario through the Ministry of Research and Innovation. The data and/or materials supporting this publication can be accessed at http://dx.doi.org/10.17630/f17227bc-3045-40d6-b289-30a4cla8966c.

\section{Author contributions}

M.H.H. S.D.E. C.K.K and K.F carried out the experiments; K.F., H.E. and S.U. synthesized and characterized the samples; M.H.H., S.D.E. and K.F. developed and carried out analysis; E.-A.K., M.J.L. and S.S. provided theoretical guidance; A.P.M. and J.C.D. supervised the project and wrote the paper with key contributions from M.H.H., S.D.E., C.K.K. and K.F. The manuscript reflects the contributions and ideas of all authors.

\section{Additional information}

Supplementary information is available in the online version of the paper. Reprints and permissions information is available online at www.nature.com/reprints. Correspondence and requests for materials should be addressed to J.C.D.

\section{Competing financial interests}

The authors declare no competing financial interests. 